\title{
ATF2 promotes urothelial cancer outgrowth via cooperation with androgen receptor signaling
}

\author{
Satoshi Inoue 1,2,3,4, Taichi Mizushima 1,2,3,4, Hiroki Ide ${ }^{3,4}$, Guiyang Jiang 1,2, Takuro Goto ${ }^{1,2}$, Yujiro Nagata ${ }^{1,2}$, \\ George J Netto ${ }^{3,4,5}$ and Hiroshi Miyamoto $1,2,3,4,6$ \\ ${ }^{1}$ Department of Pathology \& Laboratory Medicine, University of Rochester Medical Center, Rochester, New York, USA \\ 2James P. Wilmot Cancer Institute, University of Rochester Medical Center, Rochester, New York, USA \\ ${ }^{3}$ Department of Pathology, Johns Hopkins University School of Medicine, Baltimore, Maryland, USA \\ ${ }^{4}$ James Buchanan Brady Urological Institute, Johns Hopkins University School of Medicine, Baltimore, Maryland, USA \\ ${ }^{5}$ Department of Pathology, University of Alabama at Birmingham, Birmingham, Alabama, USA \\ ${ }^{6}$ Department of Urology, University of Rochester Medical Center, Rochester, New York, USA
}

Correspondence should be addressed to H Miyamoto: hiroshi_miyamoto@urmc.rochester.edu

\begin{abstract}
We investigated the functional role of ATF2, a transcription factor normally activated via its phosphorylation in response to phospho-ERK/MAPK signals, in the outgrowth of urothelial cancer. In both neoplastic and non-neoplastic urothelial cells, the expression levels of androgen receptor (AR) correlated with those of phospho-ATF2. Dihydrotestosterone treatment in AR-positive bladder cancer cells also induced the expression of phospho-ATF2 and phospho-ERK as well as nuclear translocation and transcriptional activity of ATF2. Meanwhile, ATF2 knockdown via shRNA resulted in significant decreases in cell viability, migration and invasion of AR-positive bladder cancer lines, but not AR-negative lines, as well as significant increases and decreases in apoptosis or G0/G1 cell cycle phase and S or G2/M phase, respectively. Additionally, the growth of AR-positive tumors expressing ATF2-shRNA in xenograft-bearing mice was retarded, compared with that of control tumors. ATF2 knockdown also resulted in significant inhibition of neoplastic transformation induced by a chemical carcinogen 3-methylcholanthrene, as well as the expression of BCl-2/cyclin-A2/cyclin-D1/JUN/MMP-2, in immortalized human normal urothelial SVHUC cells stably expressing $A R$, but not AR-negative SVHUC cells. Finally, immunohistochemistry in surgical specimens demonstrated significant elevation of ATF2/phospho-ATF2/phospho-ERK expression in bladder tumors, compared with non-neoplastic urothelial tissues. Multivariate analysis further showed that moderate/strong ATF2 expression and phospho-ATF2 positivity were independent predictors for recurrence of low-grade tumors (hazard ratio $(H R)=2.956$, $P=0.045)$ and cancer-specific mortality of muscle-invasive tumors ( $H R=5.317, P=0.012)$, respectively. Thus, ATF2 appears to be activated in urothelial cells through the AR pathway and promotes the development and progression of urothelial cancer.
\end{abstract}

Endocrine Connections (2018) 7, 1397-1408

\section{Introduction}

Urinary bladder cancer, generally urothelial carcinoma that predominantly affects males throughout the world (1), remains a major public health issue. More than two-thirds of patients initially present with non-muscle-invasive disease and suffer from tumor recurrence following transurethral resection and currently available
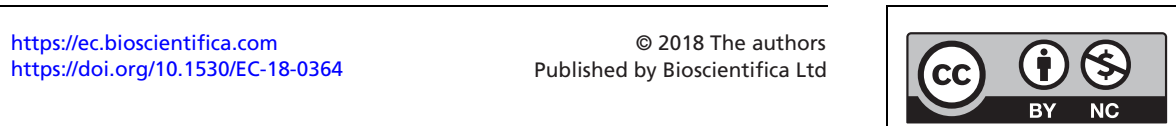
intravesical pharmacotherapy. Meanwhile, muscleinvasive bladder cancer is associated with a high risk of disease progression even after more aggressive treatment such as radical cystectomy with neoadjuvant/adjuvant chemotherapy. Accordingly, identifying key molecules/ pathways responsible for the development and/or growth of urothelial tumor may offer novel targeted therapy that improves patient outcomes.

Emerging evidence has suggested a vital role of androgen-mediated androgen receptor (AR) signals in the induction of urothelial tumorigenesis and tumor progression (reviewed in (2)), which may partly explain the gender disparity in the incidence of bladder cancer. Using preclinical models, AR inactivation has thus been shown to lead to the inhibition of bladder cancer outgrowth. We have additionally demonstrated that AR activation correlates with resistance to cisplatin treatment (3) and radiotherapy (4) in bladder cancer cells. However, the underlying mechanism of how AR and related signals modulate bladder carcinogenesis and tumor cell growth remains largely unknown.

ATF2 (activating transcription factor 2) is a member of the activating protein-1 (AP1) transcription factor family that functions via homo- or heterodimerization within family members, such as Jun and Fos $(5,6)$. It has been characterized as a mediator of not only cellular responses to stimuli, such as stresses, but also oncogenesis and progression of several types of neoplasms. Previous studies have also indicated that ATF2 can be phosphorylated by various kinases, including extracellular signal-regulated kinase (ERK) on Thr71 (7).

We previously demonstrated that dihydrotestosterone (DHT), especially in the presence of epidermal growth factor (EGF), could activate the EGF receptor (EGFR)/ ERBB2/ERK pathway in AR-positive bladder cancer cells (8). We therefore anticipated that AR signals in bladder cancer cells contributed to the modulation of ATF2 activity. Indeed, in a profiling array we previously performed (9), ATF2 was one of transcription factors that demonstrated substantial changes in their expression by androgen treatment in UMUC3 bladder cancer cells. In the present study, we investigated the role of ATF2 in urothelial tumorigenesis and tumor progression in relation to AR signaling. We found that ATF2 activity correlated with considerable induction of neoplastic transformation of AR-positive urothelial cells and the growth of AR-positive bladder cancer cells, but not those of AR-negative cells.

https://ec.bioscientifica.com

https://doi.org/10.1530/EC-18-0364 (c) 2018 The authors Published by Bioscientifica Ltd

\section{Materials and methods}

\section{Antibodies and chemicals}

We purchased anti-AR (N-20), anti-GAPDH (6c5) and anti-Histone H1 (FL-219) antibodies and anti-ATF2 (C-19), anti-p-ATF2 (Thr71) and anti-p-p44/42 MAPK (ERK) (Thr202/Thr204) antibodies from Santa Cruz Biotechnology and Cell Signaling Technology, respectively. We obtained DHT and hydroxyflutamide (HF) from Sigma-Aldrich. C6 ceramide and SCH 772984 were from Cayman Chemical.

\section{Cell lines}

Human urothelial carcinoma cell lines (UMUC3 and 5637) and an immortalized human normal urothelial cell line (SVHUC) were originally obtained from the American Type Culture Collection. Another human urothelial carcinoma cell line $(647 \mathrm{~V})$ was used in our previous studies $(3,4,9,10,11,12)$. All these lines were recently authenticated, using GenePrint 10 System (Promega), and routinely tested for Mycoplasma contamination, using PCR Mycoplasma Detection Kit (Applied Biological Materials). Stable sublines, including UMUC3-controlshRNA/UMUC3-AR-shRNA (8), 647V-vector/647V-AR (9) and SVHUC-vector/SVHUC-AR (13), were established in our previous studies. Similarly, ATF2-shRNA lentiviral particles (sc-29205-V, Santa Cruz Biotechnology) were stably expressed in UMUC3, 647V-AR, 647V, SVHUC-AR and SVHUC cells. Multiple frozen aliquots were made upon the acquisition and all experiments were performed with cells undergoing fewer than 20 passages. UMUC3/647V/5637 (or their sublines) and SVHUC (or its sublines) were maintained in Dulbecco's modified Eagle's medium (Mediatech) and Ham's F-12K (Kaighn's) medium (Mediatech), respectively, supplemented with $10 \%$ fetal bovine serum (FBS) and cultured in phenol red-free medium supplemented with either 5\% regular FBS or 5\% charcoal-stripped FBS (for DHT treatment) at least $24 \mathrm{~h}$ before experimental treatment.

\section{Western blot}

Proteins (30-50 $\mu$ g) obtained from cell extracts were separated in $10 \%$ SDS-PAGE, transferred to PVDF membrane electronically, blocked and incubated with an appropriate dilution of each specific antibody and a secondary antibody (anti-mouse IgG HRP-linked antibody or anti-rabbit IgG HRP-linked antibody; Cell Signaling 
Technology) followed by scanning with an imaging system (ChemiDOC MP, Bio-Rad). We also used a nuclear and cytoplasmic extraction reagent kit (NE-PER, Thermo Scientific) for obtaining separate nuclear/cytoplasmic fractions.

\section{Immunofluorescent staining}

Cells plated onto eight-well chamber slides (NuncLab-Tek, Thermo Scientific) were cultured in medium containing ethanol, DHT and/or HF for $24 \mathrm{~h}$. At the end of the drug treatment, the adherent cells were rinsed and fixed by $4 \%$ paraformaldehyde. The cells were then blocked with $1 \%$ bovine serum albumin for $1 \mathrm{~h}$ at $37^{\circ} \mathrm{C}$, and an antiATF2 antibody (dilution 1:50) was added and incubated for $1 \mathrm{~h}$ at $37^{\circ} \mathrm{C}$. Fluorescence images were acquired with a fluorescence microscopy (EVOS FL Auto, Life Technologies).

\section{Reporter gene assay}

Cells at a density of 50-70\% confluence in 24-well tissue culture plates were co-transfected with $250 \mathrm{ng}$ of an AP1 reporter plasmid DNA (LR-2006, Signosis) and 2.5 ng of a control reporter plasmid (pRL-CMV), using Lipofectamine 3000 transfection reagent (Life Technologies). After transfection, the cells were cultured in the presence or absence of DHT and/or HF for $24 \mathrm{~h}$. Cell lysates were then assayed for luciferase activity measured using a Dual-Luciferase Reporter Assay kit (Promega).

\section{MTT assay}

Cells (500-1000/well) seeded in 96-well tissue culture plates were cultured for 72-96h and then incubated with $0.5 \mathrm{mg} / \mathrm{mL}$ of MTT (Sigma-Aldrich) in $100 \mu \mathrm{L}$ of medium for $3 \mathrm{~h}$ at $37^{\circ} \mathrm{C}$. MTT was dissolved by DMSO, and the absorbance was measured at a wavelength of $570 \mathrm{~nm}$ with background subtraction at $630 \mathrm{~nm}$.

\section{Plate colony formation assay}

Cells (500/well) seeded in 12-well tissue culture plates were allowed to grow until colonies in the control well were easily distinguishable. The cells were then fixed with methanol and stained with $0.1 \%$ crystal violet. The number of colonies in photographed pictures was quantitated, using ImageJ software (National Institutes of Health).

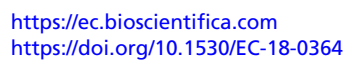

\section{Apoptosis and cell cycle analysis}

The TUNEL assay was performed on cell-burdening coverslips, using the DeadEnd Fluorometric TUNEL system (Promega), followed by counterstaining for DNA with 4',6'-diamidino-2-phenylindole (DAPI). Apoptotic index was determined in the cells visualized by the fluorescence microscopy (EVOS FL Auto). For cell cycle phase quantification, a kit (Cell Cycle Assay Cell-Clock biocolor) was used according to the manufacturer's protocol. Data were analyzed, using ImageJ software.

\section{Scratch wound-healing assay}

Cells at a density of $\geq 90 \%$ confluence in 12 -well tissue culture plates were scratched manually with a sterile $200 \mu \mathrm{L}$ plastic pipette tip, cultured for $24 \mathrm{~h}$, fixed with methanol and stained with $0.1 \%$ crystal violet. The width of the wound area was monitored with an inverted microscope, and the normalized cell-free area in photographed pictures ( $24 \mathrm{~h} / 0 \mathrm{~h})$ was quantitated, using ImageJ software.

\section{Cell invasion assay}

Cells $\left(1 \times 10^{5}\right)$ in $500 \mu \mathrm{L}$ of serum-free medium were added to the upper chamber of a Matrigel-coated transwell chamber $(8.0 \mu \mathrm{m}$ pore size polycarbonate filter with $6.5 \mathrm{~mm}$ diameter; Corning), whereas $750 \mu \mathrm{L}$ of medium containing 10\% FBS was added to the lower chamber of the transwell. After incubation for $16 \mathrm{~h}$, invaded cells were fixed, stained with $0.1 \%$ crystal violet and counted.

\section{In vitro transformation}

An in vitro neoplastic/malignant neoplastic transformation system was employed, using SVHUC line upon exposure to a carcinogen 3-methylcholanthrene (MCA), as established in a previous study (14), with minor modifications. Briefly, cells $\left(2 \times 10^{6} / 10 \mathrm{~cm}\right.$ culture dish incubated for $24 \mathrm{~h}$ ) were cultured in serum-free F-12K containing $5 \mu \mathrm{g} / \mathrm{mL}$ MCA (Sigma-Aldrich). After the first $24 \mathrm{~h}$ of MCA exposure, 1\% FBS was added to the medium. After additional $24 \mathrm{~h}$ of MCA exposure, the cells were cultured in medium containing 5\% FBS (without MCA) until near confluence. Subcultured cells (1:3 split ratio) were again incubated with MCA for two 48-hour exposure periods, using the above protocol. These MCA-exposed cells were subcultured for 6 weeks and then utilized for subsequent assays.

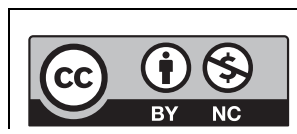

This work is licensed under a Creative Commons Attribution-NonCommercial 4.0 International License. 


\section{Reverse transcription (RT) and real-time PCR}

Total RNA isolated from cultured cells by TRIzol (Invitrogen) was reverse transcribed, using oligo-dT primers and Ominiscript reverse transcriptase (Qiagen). Real-time PCR was then performed, using RT2 SYBR Green FAST Mastermix (Qiagen). The primer sequences are given in Supplementary Table 1 (see section on supplementary data given at the end of this article).

\section{Animal models}

The animal protocol used in this study was approved by the University Committee on Animal Resources at the University of Rochester (protocol number: 2017007/101860).

SVHUC- or UMUC3-derived cells $\left(1 \times 10^{6}\right)$ mixed with $100 \mu \mathrm{L}$ Matrigel (BD Biosciences) were subcutaneously injected into the flank of 6-week-old male immunocompromised athymic NCr-nu/nu mice (National Cancer Institute). Tumor size was estimated by serial caliper measurements of perpendicular diameters using the following formula ((short diameter $)^{2} \times$ (longest diameter) $\times 0.5)$.

\section{Tissue microarray (TMA) and} immunohistochemistry (IHC)

A set of TMA consisting of retrieved bladder tissue specimens obtained by transurethral resection performed at the Johns Hopkins Hospital was constructed previously upon appropriate approval from the institutional review board. The TMA in which AR was immunohistochemically stained in our previous study (15) consisted of 129 cases of primary urothelial neoplasm from 98 men and 31 women with a mean/median age of 65.7/69 years (range: 26-89). All 51 patients with muscle-invasive tumor ultimately underwent radical cystectomy. None of the patients had received therapy with radiation or anti-cancer drugs prior to the collection of the tissues.

IHC was performed on the $5 \mu \mathrm{m}$ sections, using a primary antibody to ATF2 (dilution 1:500), p-ATF2 (dilution 1:250) or p-ERK (dilution 1:1000), as we described previously $(8,11)$. All stains were manually quantified by two pathologists ( $\mathrm{G}$ \& $\mathrm{H} \mathrm{M}$ ) who were blinded to sample identity. The German immunoreactive scores calculated by multiplying the percentage $(0 \%=0 ; 1-10 \%=1$; $11-50 \%=2 ; 51-80 \%=3 ; 81-100 \%=4$ ) of immunoreactive cells in their nuclei by staining intensity (negative $=0$; weak $=1 ; \quad$ moderate $=2 ; \quad$ strong $=3$ ) were considered

https://ec.bioscientifica.com

https://doi.org/10.1530/EC-18-0364 (c) 2018 The authors Published by Bioscientifica Ltd negative $(0 ; 0-1)$, weakly positive $(1+; 2-4)$, moderately positive $(2+; 6-8)$ and strongly positive $(3+; 9-12)$.

\section{Statistical analysis}

At least three independent experiments were performed in cell line assays. Fisher's exact test and Student's $t$-test/ Mann-Whitney $U$ test were used to assess statistical significance for categorized variables and those with a continuous distribution, respectively. Correlations between variables were determined by the Spearman's correlation coefficient (CC). The rates of xenograft tumor formation and patient survival were calculated by the Kaplan-Meier method and comparison was made by log-rank test. The Cox proportional hazards model was used to determine statistical significance of predictors in a multivariate setting. $P$ values less than 0.05 were considered statistically significant.

\section{Results}

Interplay between ATF2 and AR signals in bladder cancer cells

We first assessed the expression of ATF2 and its active form p-ATF2 in urothelial cell lines. Western blot showed that the levels of ATF2/p-ATF2 expression were higher in UMUC3 than in other bladder cancer lines, 5637 and $647 \mathrm{~V}$, and the weakest in a normal urothelial line SVHUC (Fig. 1A). We next investigated the impact of AR knockdown/overexpression and androgen treatment on ATF2/p-ATF2 expression. In UMUC3/647V/SVHUC cells, AR expression was found to correlate with that of p-ATF2, but not ATF2 (Fig. 1B). Similarly, in AR-positive cancer cells, DHT treatment induced the expression of p-ATF2, which was blocked by an anti-androgen HF, but not that of ATF2 (Fig. 1C). When subcellular localization of ATF2 was examined by western blot, DHT treatment resulted in increases and decreases in nuclear and cytoplasmic ATF2 expression, respectively, and HF antagonized the androgen effects (Fig. 1D). Promotion of nuclear translocation of ATF2 by androgen was further confirmed by immunofluorescence (Fig. 1E and Supplementary Fig. 1A). ATF2-mediated transcriptional activity was then determined in the cell extracts with transfection of an AP1 luciferase reporter plasmid and subsequent treatment with androgen and/or anti-androgen. DHT considerably augmented ATF2 luciferase activity, compared with mock treatment, and HF restored the enhancement (Fig. 1F). 
A

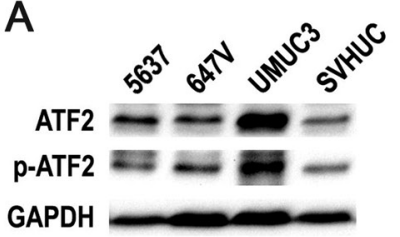

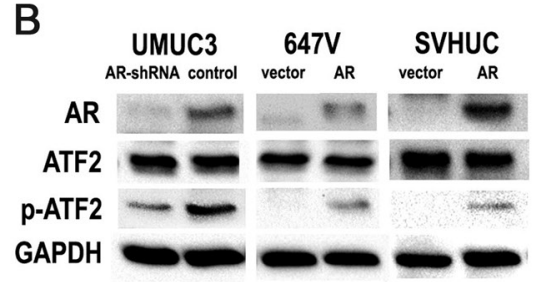

C

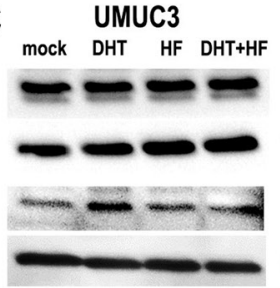

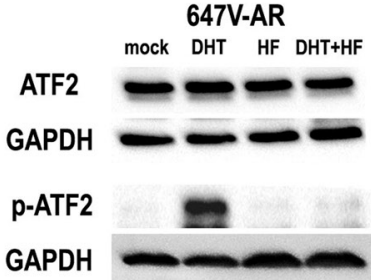

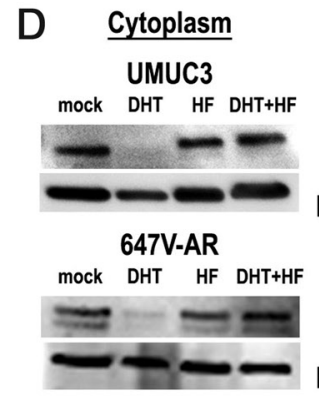

G

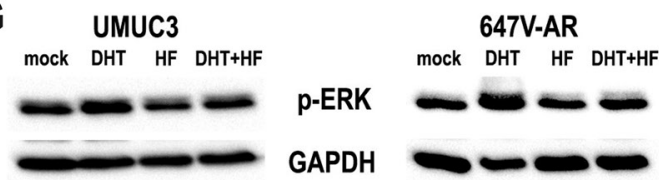

UMUC3 GAPDH/
Histone H1

ATF2

GAPDH/ Histone $\mathrm{H} 1$

D. 20
E

mock DHT HF DHT+HF

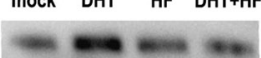

mock DHT HF DHT+HF

en $-4=0$

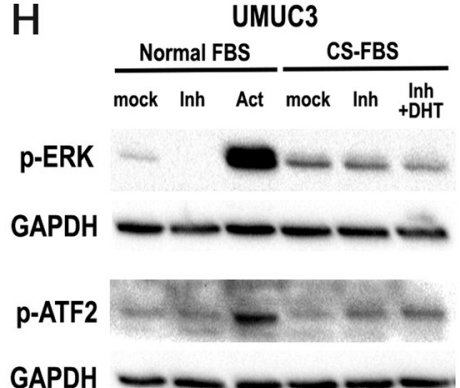

GAPDH
F

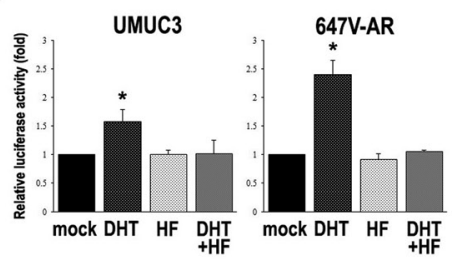

mock DHT HF DHT
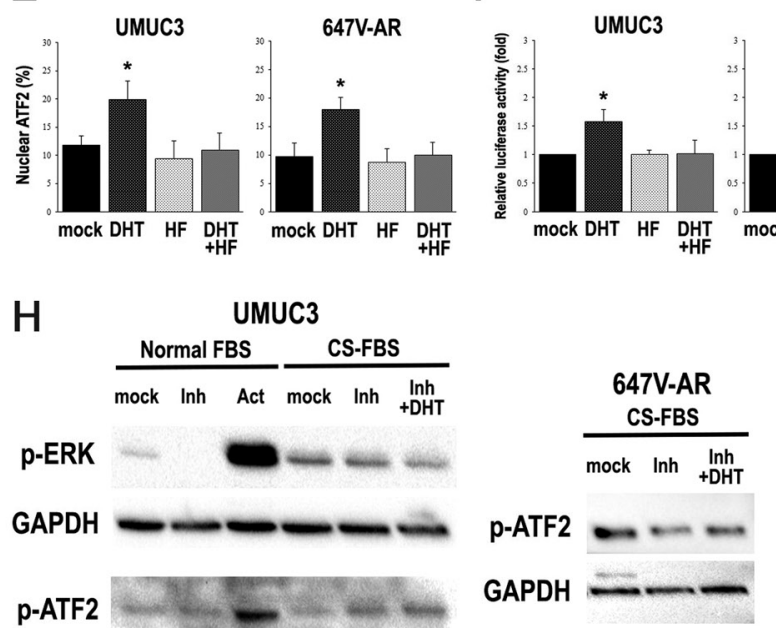

\section{Figure 1}

Effects of androgen on ATF2 expression/activity in bladder cancer cells. (A) Western blotting of ATF2 and p-ATF2 in 5637/647V/UMUC3/SVHUC cells. (B) Western blotting of AR, ATF2 and p-ATF2 in UMUC3-AR-shRNA/control-shRNA, 647V-vector/AR and SVHUC-vector/AR cells. Western blotting of ATF2 and p-ATF2, using total proteins (C) or separate nuclear and cytoplasmic protein fractions (D) extracted from UMUC3 or 647V-AR cells cultured with ethanol (mock), DHT (10 nM) and/or HF $(5 \mu \mathrm{M})$ for $24 \mathrm{~h}$. GAPDH and Histone $\mathrm{H} 1$ served as internal controls of total/nuclear and cytoplasmic proteins, respectively. Immunofluorescent staining (E) and luciferase reporter activity (F) of ATF2 in UMUC3 or 647V-AR cells cultured with ethanol (mock), DHT (10 nM) and/or $\mathrm{HF}(5 \mu \mathrm{M})$ for $24 \mathrm{~h}$. Each value presented relative to that of mock treatment represents the mean (+S.D.) from three independent experiments. ${ }^{*} P<0.05$ (vs mock treatment). (G) Western blotting of p-ERK in UMUC3 or $647 \mathrm{~V}-\mathrm{AR}$ cells cultured with ethanol (mock), DHT (10 nM) and/or HF (5 $\mu \mathrm{M})$ for $24 \mathrm{~h}$. (H) Western blotting of p-ERK and/or p-ATF2 in UMUC3 or 647V-AR cells pretreated with SCH 772984 ( $2 \mu \mathrm{M}$ for $6 \mathrm{~h}$; 'Inh') or C6 ceramide ( $50 \mu \mathrm{M}$ for $24 \mathrm{~h}$; 'Act') with (in media containing charcoal-stripped FBS (CS-FBS)) or without (normal FBS) additional treatment of ethanol (mock) or DHT (10 nM) for $24 \mathrm{~h}$.

These results suggest that androgen activates ATF2 signals by inducing its nuclear translocation.

We previously demonstrated that androgen induced the activity of MAPK in bladder cancer cells (8), and ATF2 was known to be a downstream target of its pathway (7). We confirmed that DHT induced the expression of p-ERK in AR-positive lines (Fig. 1G). An ERK activator also induced the expression of p-ATF2 (Fig. 1H). Additionally, in the presence of an ERK inhibitor, DHT did not significantly induce the expression of p-ERK as well as p-ATF2.

\section{Role of ATF2 in bladder cancer progression}

To investigate the functional role of ATF2 in cell proliferation, migration and invasion of bladder cancer, an ATF2-shRNA was stably expressed in AR-positive and
AR-negative bladder cancer lines where ATF2 knockdown did not significantly affect AR expression (Fig. 2A). We compared cell viability via MTT assay (Fig. 2B) and colony formation via clonogenic assay (Fig. 2C) between ATF2positive lines vs their knockdown lines. ATF2 knockdown resulted in significant decreases in the cell/colony number of AR-positive lines. By contrast, ATF2 knockdown did not significantly affect the growth of AR-negative cells. TUNEL (Fig. 2D and Supplementary Fig. 1B) and cell cycle (Fig. 2E and Supplementary Fig. 1C) assays in AR-positive/ ATF2-knockdown lines further showed significant increases in apoptosis as well as the G0/G1 phase and significant decreases in the $\mathrm{S}$ and $\mathrm{G} 2 / \mathrm{M}$ phases, compared with respective control lines. We also performed a scratch wound-healing assay (Fig. 2F) and a transwell invasion assay (Fig. 2G) to compare cell migration and https://ec.bioscientifica.com

https://doi.org/10.1530/EC-18-0364
C 2018 The authors Published by Bioscientifica Ltd

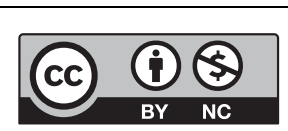

This work is licensed under a Creative Commons Attribution-NonCommercial 4.0 International License. 


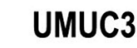

control ATF2 -shRNA
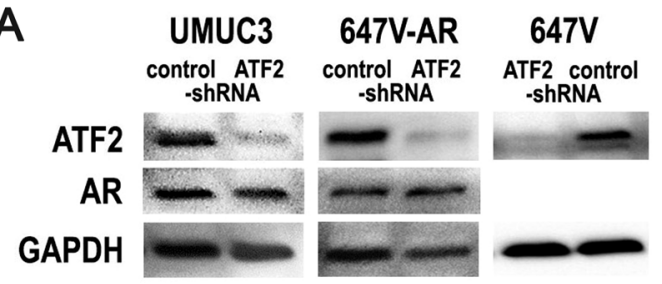

$\mathrm{H}$

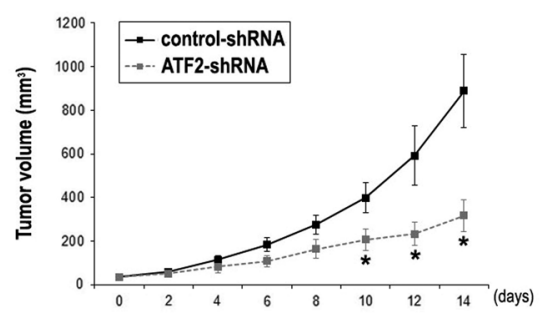

B
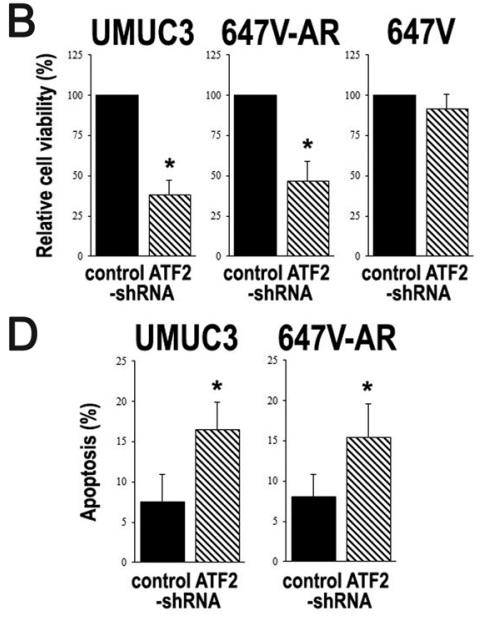

F

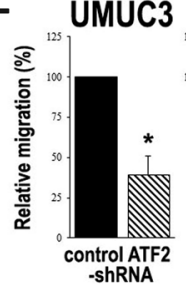

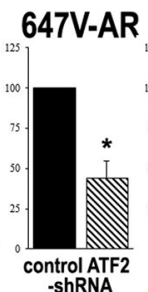

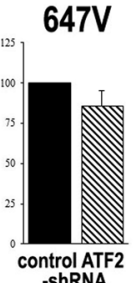

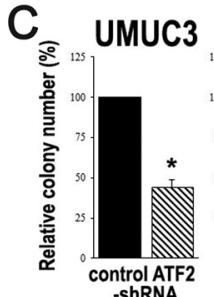

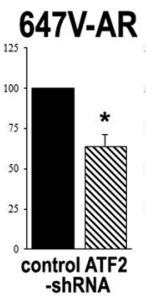

$647 \mathrm{~V}$

$E$

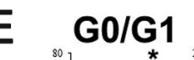

$\mathbf{S}$
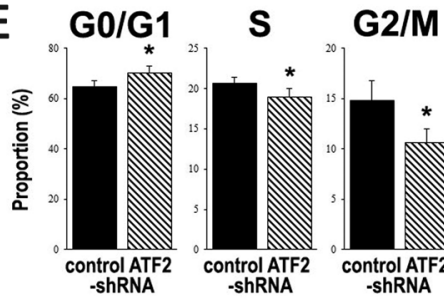

G
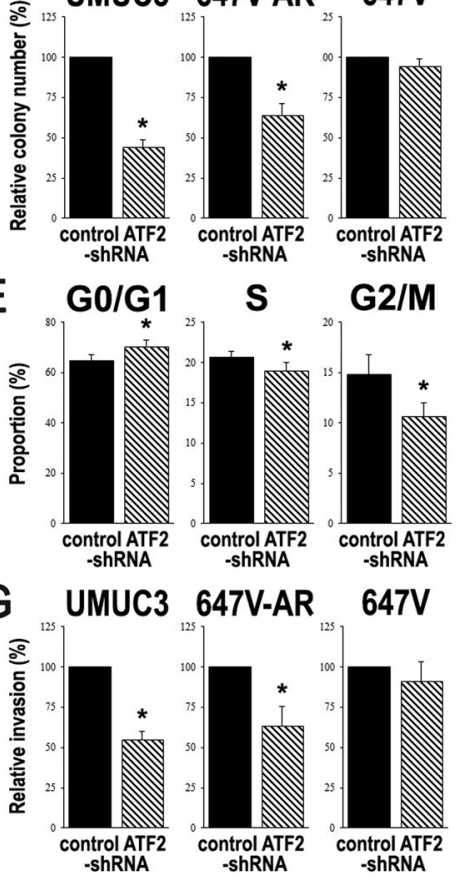

Figure 2

Effects of ATF2 knockdown on bladder cancer outgrowth. (A) Western blotting of ATF2 and AR in UMUC3/647V-AR/SVHUC-control-shRNA vs UMUC3/647V-AR/SVHUC-ATF2-shRNA. (B) MTT assay in UMUC3/647V-AR/647V-control-shRNA vs UMUC3/647V-AR/647V-ATF2-shRNA cultured for 72h. (C) Clonogenic assay in UMUC3/647V-AR/647V-control-shRNA vs UMUC3/647V-AR/647V-ATF2-shRNA cultured for 2 weeks. The number of colonies consisting of $\geq 20$ cells was counted. (D) TUNEL assay in UMUC3/647V-AR-control-shRNA vs UMUC3/647V-AR-ATF2-shRNA. (E) Cell cycle phase analysis in UMUC3control-shRNA vs UMUC3-ATF2-shRNA. (F) Wound-healing assay in UMUC3/647V-AR/647V-control-shRNA vs UMUC3/647V-AR/647V-ATF2-shRNA. The cells grown to confluence were gently scratched, and the wound area was measured after 24-h culture. The migration was determined by the rate of cells filling the wound area. (G) Transwell invasion assay in UMUC3/647V-AR/647V-control-shRNA vs UMUC3/647V-AR/647V-ATF2-shRNA. The number of invaded cells present in the lower chamber was counted under a light microscope (100x objective in five random fields). Each value presented relative to that of control-shRNA subline (except apoptotic index) represents the mean (+s.D.) from three independent experiments. ${ }^{*}<<0.05$ (vs control-shRNA). (H) UMUC3-control-shRNA/ATF2-shRNA cells were subcutaneously implanted into the flank of athymic NCr-nu/nu mice ( $n=9 / \mathrm{group}$ ). Day 0 was set as estimated volume of each tumor exceeding $30 \mathrm{~mm}^{3}$. Each value represents the mean (+S.D.). ${ }^{*} P<0.05$ (vs control-shRNA at each day).

invasion, respectively. Similar to the above findings, ATF2 knockdown demonstrated marked decreases in the migration/invasion ability of AR-positive cells, but not in that of AR-negative cells.

Next, we used mouse xenograft models to assess the effects of ATF2 knockdown on bladder tumor outgrowth in vivo. UMUC3-control-shRNA/UMUC3-ATF2-shRNA cells were implanted subcutaneously into the flank of immunocompromised mice, and tumor size was monitored following its formation (i.e. day 0 when the estimated tumor volume reached $30 \mathrm{~mm}^{3}$ ). The inoculated ATF2-shRNA tumors were found to be smaller than controlshRNA tumors, especially at day 10 or after (Fig. 2H).

\section{Role of ATF2 in urothelial tumorigenesis}

To investigate the functional role of ATF2 in urothelial tumorigenesis, we used an in vitro transformation model where non-neoplastic SHVUC cells could undergo

$\begin{array}{lr}\text { https://ec.bioscientifica.com } & \text { () } 2018 \text { The authors } \\ \text { https://doi.org/10.1530/EC-18-0364 } & \text { Published by Bioscientifica Ltd }\end{array}$

stepwise transformation upon exposure to a chemical carcinogen MCA (14). SVHUC-AR and SVHUC stably expressing either a control-shRNA or an ATF2-shRNA (Fig. 3A) were exposed to MCA and subsequently cultured for 6 weeks. The levels of AR expression were similar between SVHUC-AR-control-shRNA and SVHUC-AR-ATF2shRNA. In addition, there were no significant differences in the rates of cell proliferation of these sublines (e.g. 12\% decrease by ATF2-shRNA in SVHUC-AR cells, $P=0.364$ ) without MCA exposure. Oncogenic activity of MCASVHUC sublines was then monitored by cell viability (MTT assay; Fig. 3B), colony formation (clonogenic assay; Fig. 3C) and tumor formation (mouse xenograft model; Fig. 3D). We thus compared the degree of neoplastic/ malignant transformation in ATF2-positive vs ATF2negative urothelial cells with the carcinogen challenge. In all the three assays, ATF2 knockdown in MCA-SVHUC-AR cells resulted in significant inhibition of their neoplastic transformation. By contrast, ATF2 knockdown did not

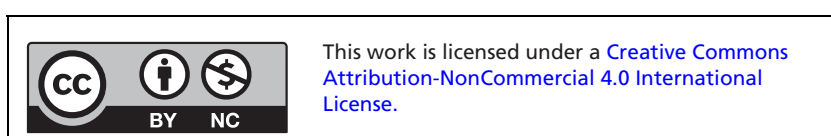


A

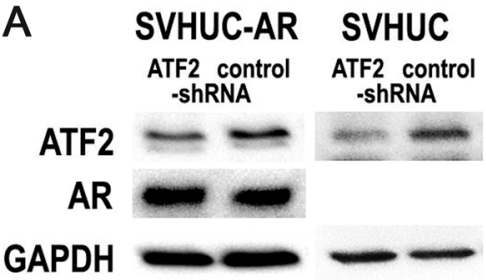

B
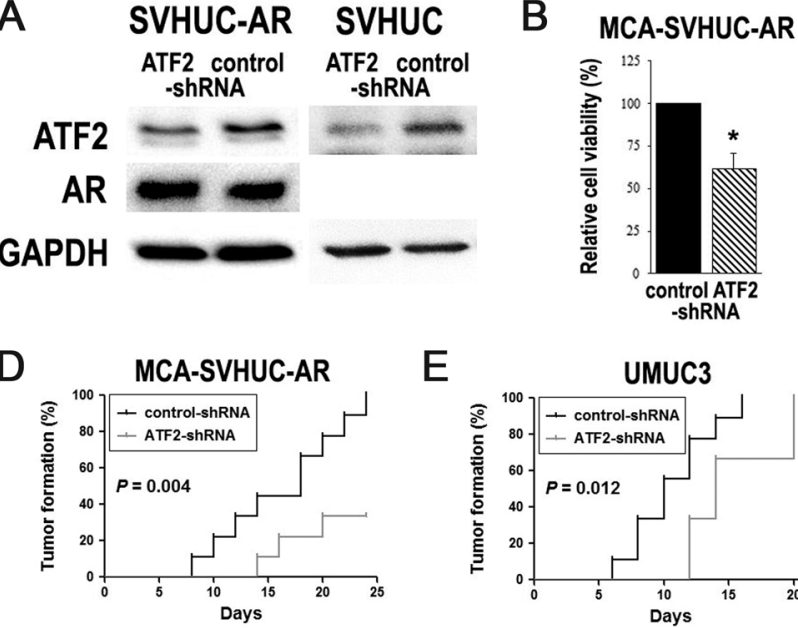

E

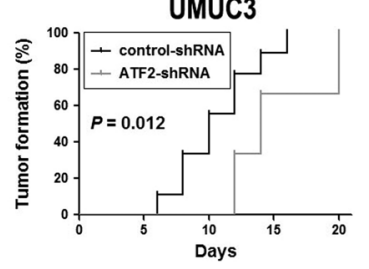

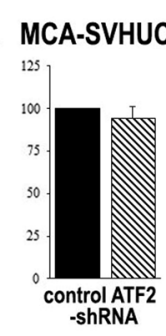

\section{Figure 3}

Effects of ATF2 knockdown on neoplastic transformation of urothelial cells. (A) Western blotting of ATF2 in SVHUC-AR/SVHUC-control-shRNA vs SVHUC-AR/SVHUC-ATF2-shRNA. SVHUC-AR/SVHUC-control-shRNA and SVHUC-AR/SVHUC-ATF2-shRNA cells exposed to MCA and subsequently cultured for 6 weeks were seeded for MTT assay (B; additional 96-h culture) or clonogenic assay (C; additional 2-week culture). Cell viability or colony number ( $\geq 20$ cells) presented relative to that in control-shRNA cells represents the mean (+s.D.) from three independent experiments. ${ }^{*} P<0.05$ (vs control-shRNA). SVHUC-AR-control-shRNA/ATF2-shRNA cells exposed to MCA and subsequently cultured for 6 weeks (D) or UMUC3-control-shRNA/ATF2-shRNA cells (E) were subcutaneously implanted into the flank of athymic $\mathrm{NCr}-\mathrm{nu} / \mathrm{nu}$ mice ( $n=9 / \mathrm{group}$ ). The endpoint for this study was tumor formation (exceeding $30 \mathrm{~mm}^{3}$ in its estimated volume or $5 \mathrm{~mm}$ in greatest dimension).

significantly have an impact on neoplastic transformation of AR-negative MCA-SVHUC cells.

Tumor formation of the UMUC3 xenografts, as an endpoint (i.e. $30 \mathrm{~mm}^{3}$ in estimated volume), used for the assessment of tumor growth (Fig. 2H) was compared between control-shRNA and ATF2-shRNA lines. In accordance with the above findings, ATF2 knockdown resulted in striking delay in the formation of xenograft tumors (Fig. 3E).

By using a quantitative RT-PCR method, we then compared the expression levels of oncogenic molecules that are known to play an important role in bladder tumorigenesis by modulating cell cycle progression and/or apoptosis in urothelial cells undergoing neoplastic transformation. In SVHUC-AR cells with MCA exposure, ATF2 knockdown significantly downregulated the expression of Bcl-2, cyclin A2, cyclin D1, JUN and MMP-2 (Fig. 4A). However, the expression levels of these genes were not significantly different between AR-negative MCA-SVHUC cells with vs without ATF2-shRNA (Fig. 4B).

\section{Expression of ATF2 in bladder cancer specimens and its prognostic significance}

Finally, we stained immunohistochemically for ATF2, p-ATF2 and p-ERK in the bladder TMAs consisting of 129 urothelial neoplasms and corresponding 85-86 non-neoplastic bladder tissues. Positive signals of these proteins were detected predominantly in the nucleus of benign/malignant urothelial cells (Fig. 5A).

Overall, ATF2/p-ATF2/p-ERK was positive in 54 (64\%; 27 (32\%) 1+, 24 (28\%) 2+, 3 (4\%) 3+)/2 (2\%; 2 (2\%) 1+)/9 (10\%; 8 (9\%) 1+, 1 (1\%) 2+) of 85 benign urothelial tissues and 108 (84\%; 33 (26\%) 1+, 63 (49\%) 2+, 12 (9\%) 3+)/41 (32\%; 41 (32\%) 1+)/33 (26\%; 23 (18\%) 1+, 8 (6\%) 2+, $2(2 \%) 3+$ ) of 129 tumors, respectively (Supplementary Tables 2, 3 and 4). Thus, the rates of their positivity ( 0 vs $1+/ 2+/ 3+$ ) were significantly higher in tumors than in benign tissues. We next analyzed the associations of their expression with the histopathological profile available for our patient cohort. The rate of moderate to strong $(0 / 1+$ vs $2+/ 3+)$ or strong $(0 / 1+/ 2+$ vs $3+)$ ATF2 expression was significantly higher in high-grade tumors (53 (67\%) or $11(14 \%))$ than in lower grade tumors $(22(44 \%)$ or $1(2 \%)$ ). Positivity (0 vs $1+/ 2+/ 3+$ ) or moderate/strong positivity $(0 / 1+$ vs $2+/ 3+)$ of ATF2 also tended to be more often seen in muscle-invasive tumors (47 (92\%) or 35 $(69 \%))$ than in non-muscle-invasive tumors $(61$ (78\%) or $40(52 \%))$. However, there were no significant associations between $\mathrm{p}$-ATF2/p-ERK expression and tumor grade or $\mathrm{pT}$ stage as well as between ATF2/p-ATF2/p-ERK expression and the status of lymph node involvement. In tumors, the expression levels of p-ATF2 vs p-ERK were correlated (all cases; $\mathrm{CC}=0.449, \quad P<0.001$ ) (Supplementary Table 5). Meanwhile, in our previous study (15), AR was immunohistochemically stained in the bladder

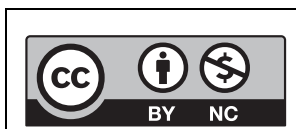

This work is licensed under a Creative Commons Attribution-NonCommercial 4.0 International License. 
A

MCA-SVHUC-AR
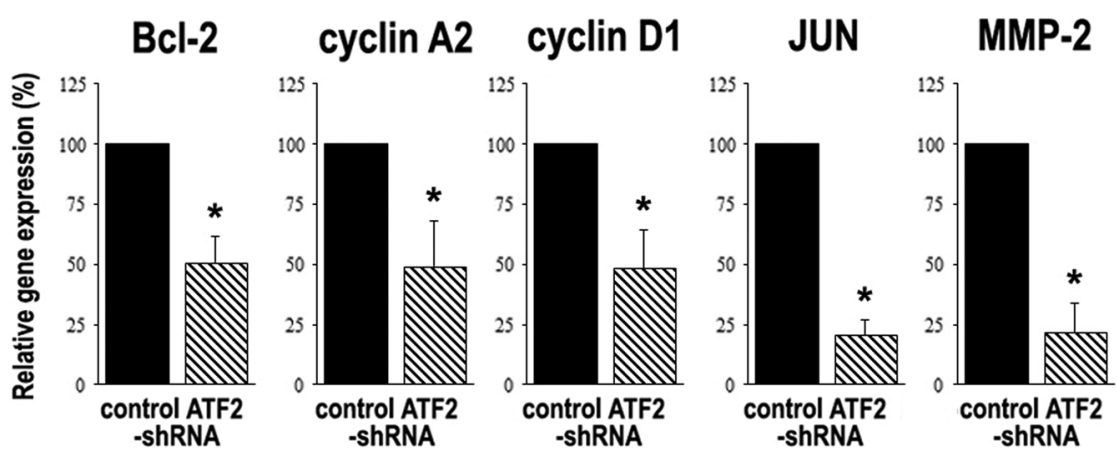

B

MCA-SVHUC
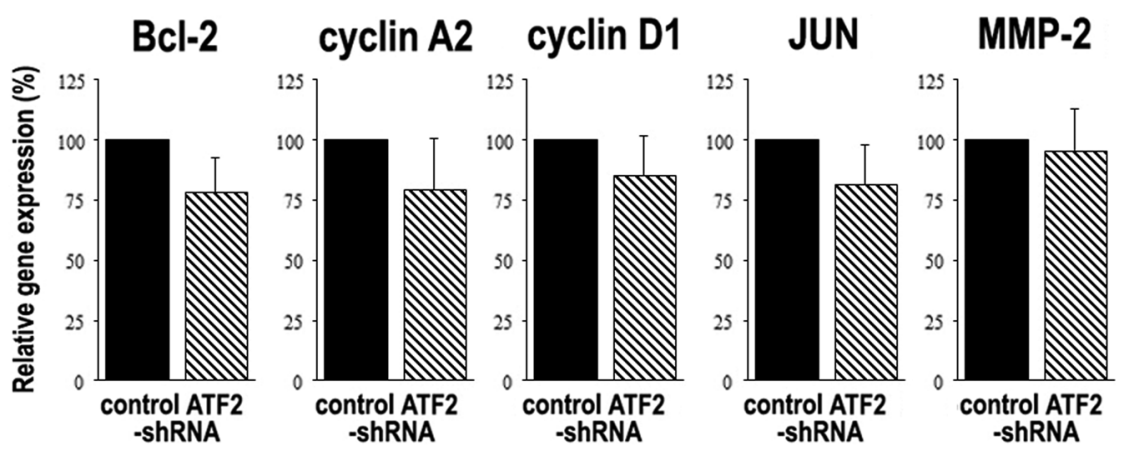

Figure 4

Effects of ATF2 knockdown on the expression of genes involving cell cycle progression and/or apoptosis in urothelial cells undergoing neoplastic transformation. SVHUC-AR-controlshRNA vs SVHUC-AR-ATF2-shRNA (A) and SVHUC-control-shRNA vs SVHUC-ATF2-shRNA (B) exposed to MCA and subsequently cultured for 6 weeks were subjected to RNA extraction and real-time RT-PCR. GAPDH was used to normalize the expression of $B C l-2$, cyclin A2, cyclin D1, JUN or $M M P-2$, which is presented relative to that of control-shRNA cells (the mean +S.D. of three independent experiments). ${ }^{\star} P<0.05$ (vs control-shRNA).

TMAs including the present 129 cases and was found to be positive in $58(45 \%)$ tumors. Remarkably, there were moderate $(0.4 \leq \mathrm{CC}<0.6)$ and weak $(0.2 \leq \mathrm{CC}<0.4)$ correlations between ATF2 and AR in high-grade non-muscle-invasive tumors and between p-ATF2 and AR in all/male/low-grade/muscle-invasive tumors, respectively.

We then performed Kaplan-Meier analysis coupled with the log-rank test to assess possible associations

\section{A ATF2}

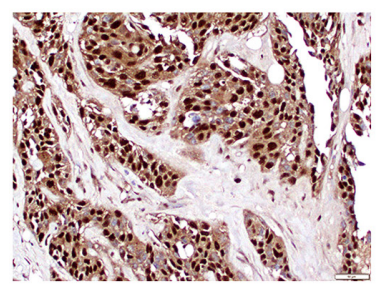

B
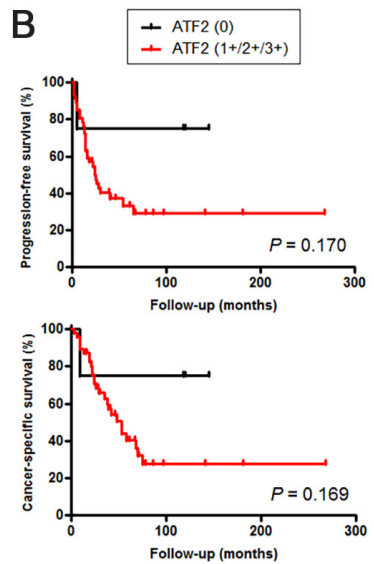

https.//ec.bioscientifica.com

https://doi.org/10.1530/EC-18-0364
p-ATF2

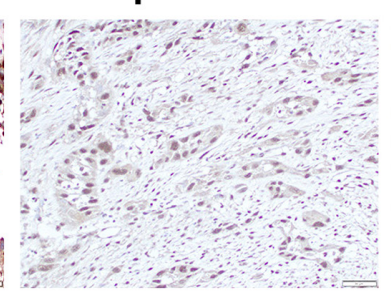

\begin{tabular}{|l|}
\hline - p-ATF2 (0) \\
+ p-ATF2 $(1+)$
\end{tabular}
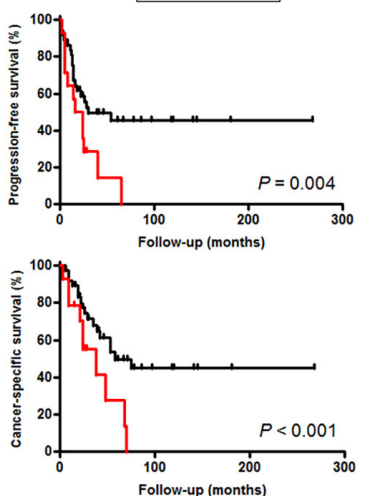
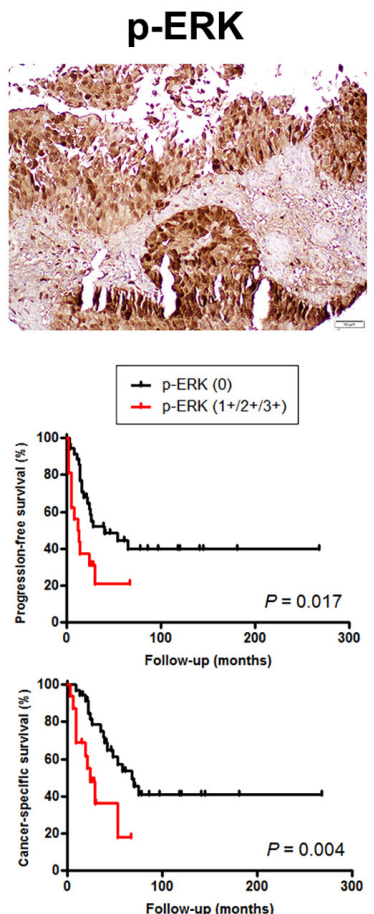

Figure 5

IHC of ATF2, p-ATF2 and p-ERK in bladder cancer specimens. (A) The staining is scored by a combination of the intensity (i.e. strong (ATF2, p-ERK), weak (p-ATF2)) and distribution (i.e. percent immunoreactive cells). Original magnification $\times 200$. (B) Kaplan-Meier analyses for PFS and CSS in patients with muscle-invasive tumor, according to ATF2/p-ATF2/p-ERK positivity.

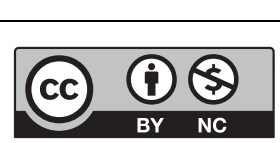

This work is licensed under a Creative Commons Attribution-NonCommercial 4.0 International License. 
between ATF2/p-ATF2/p-ERK expression and patient outcomes. There were no significant associations between the status of ATF2/p-ATF2/p-ERK expression in nonmuscle-invasive tumors and a recurrence-free survival or progression-free survival (PFS) rate as well as between that of ATF2 expression in muscle-invasive tumors and a PFS or cancer-specific survival (CSS) rate (Fig. 5B). However, patients with p-ATF2-positive/p-ERK-positive muscleinvasive tumor had significantly higher risks for disease progression $(P=0.004 / P=0.017)$ and cancer-specific mortality $(P<0.001 / P=0.004)$. Moreover, as suggested $(P=0.071)$ in our previous study involving 91 muscleinvasive tumors (15), AR positivity was significantly $(P=0.013)$ associated with disease progression in the 51 muscle-invasive tumors (Table 1 ). To determine whether ATF2/p-ATF2/p-ERK expression was an independent prognosticator, multivariate analysis was performed with Cox model (Table 1). In muscle-invasive tumors, p-ATF2 positivity was strongly associated with a lower CSS rate (HR=5.317, $P=0.012$. p-ERK positivity also showed a trend toward significance for disease progression ( $\mathrm{HR}=2.727, P=0.066)$. Additionally, in 50 cases of low grade tumors, moderate/strong expression of ATF2 was strongly associated with the risk of tumor recurrence in both univariate $(P=0.034)$ and multivariate $(\mathrm{HR}=2.956$, $P=0.045)$ settings.

\section{Discussion}

The functional role of ATF2 in urothelial tumorigenesis and cancer progression remains poorly understood, while the involvement of androgen-mediated AR signals in them has been well documented. In our previous study, we demonstrated that androgen increased the expression of p-ERK and activated the EGFR/ERBB2/ERK pathway in bladder cancer cells (8). In the current study, we first showed the activation of ATF2, as a downstream target of the ERK, through the AR pathway in bladder cancer cells. AR activation was then found to lead to considerable induction in the expression of p-ATF2, but not that of ATF2, as well as nuclear translocation and transactivation of ATF2. Furthermore, when an ERK inhibitor was pretreated, androgen failed to significantly induce p-ATF2 expression. These findings suggest that AR signals modulate ATF2 activity through the EGFR-ERK pathway in bladder cancer cells.

ATF2 is known to regulate the expression of its target genes, including those involving apoptosis, cell proliferation and DNA damage response $(6,16)$.
Accordingly, ATF2 activation has been correlated with the growth of some neoplasms, such as spindle cell carcinoma of the skin (17), extramammary Paget's disease (18), head and neck squamous cell carcinoma (19), synovial sarcoma (20) and pancreatic cancer (21). Interestingly, loss of ATF2 function has also been observed in other malignancies, such as breast and lung carcinomas as well as neuroblastoma (22), implying that the balance of tissue-specific compositions of ATF2-AP1 dimers is critical for transcriptional programs for cell survival vs cell death (6). We here demonstrated that ATF2 knockdown significantly reduced cell proliferation by modulating apoptosis and cell cycle, as well as cell migration/invasion and the growth of inoculated xenografts in mice, of bladder cancer lines possessing a functional AR.

Similar to the roles of ATF2 in tumor progression described above, ATF2 has been shown to function as both an oncogene and a tumor suppressor in a cell- or tissue-dependent manner and induce/suppress tumor development. Even for mammary tumorigenesis, both stimulation (23) and inhibition (24) by ATF2 have been documented. Dual functions of ATF2 have also been demonstrated in the development of melanoma (25, 26). Additionally, in SVHUC normal urothelial cells, treatment with arsenic, a bladder carcinogen, induced the expression of ATF2 and p-ATF2, which was inhibited by JNK or p38 inhibitors (27). We here demonstrated further evidence, using an in vitro transformation model, indicating that ATF2 could promote urothelial tumorigenesis in the presence of AR. In SVHUC-AR cells with carcinogen challenge, ATF2 knockdown resulted in significant decreases in the expression levels of oncogenic molecules. It still needs to be determined how ATF2 signaling regulates urothelial cancer initiation.

More importantly, functional interplay between ATF2 and AR signals, as documented in skeletal muscle cells (28), was confirmed in urothelial cells. Specifically, ATF2 significantly regulated neoplastic transformation of urothelial cells, as well as bladder cancer cell growth, only in the presence of AR. Thus, activated AR appears to be required for the modulation of urothelial tumorigenesis and tumor progression by ATF2. It should be determined whether AR signals are critical for the ATF2-regulated outgrowth of various malignancies in which they are known to play both significant and insignificant roles.

Several immunohistochemical studies have determined the expression levels of ATF2, p-ATF2 and/ or p-ERK in human tumor specimens. In particular, ATF2 and/or p-ATF2 were found to be significantly elevated in pancreatic cancer (21), prostate cancer (29) and spindle 


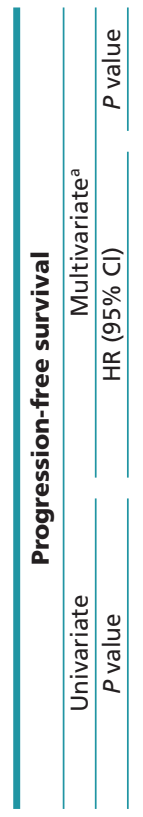

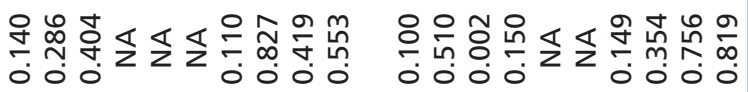

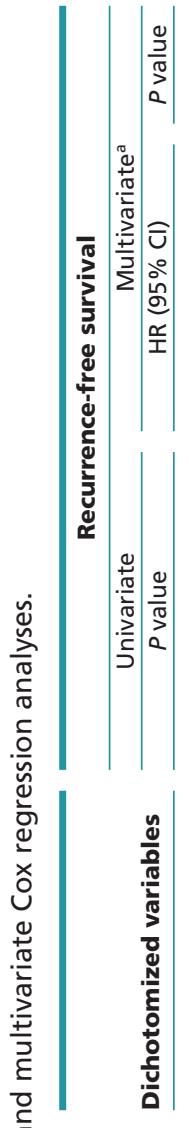

$\stackrel{\infty}{\circ}$

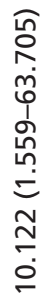

$\stackrel{n}{0}$

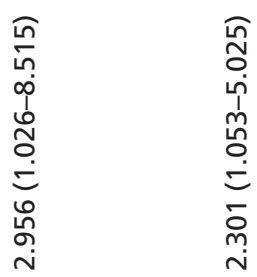

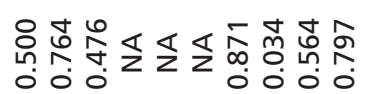

$\stackrel{\frac{0}{\pi}}{\stackrel{0}{\infty} \frac{0}{\pi}}$

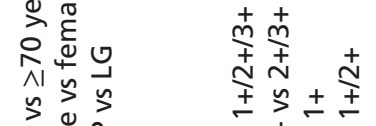

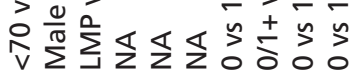

ồ.

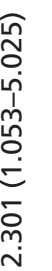

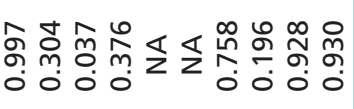

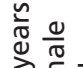

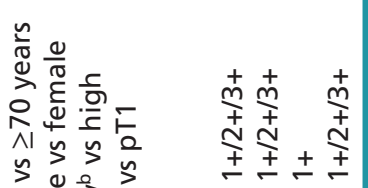

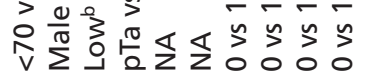
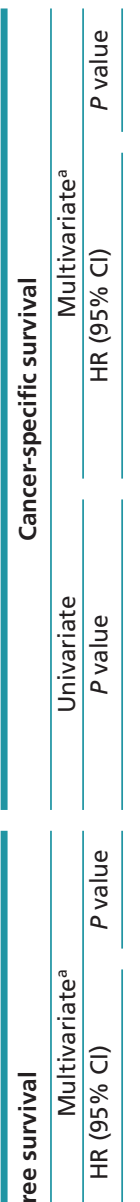

寽

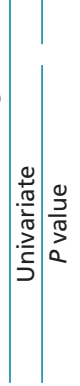

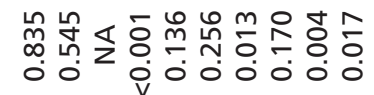

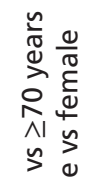

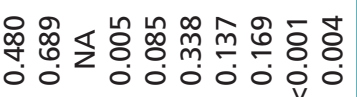

$\bar{\circ}$

$\stackrel{\bar{\tau}}{\circ}$

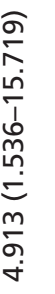

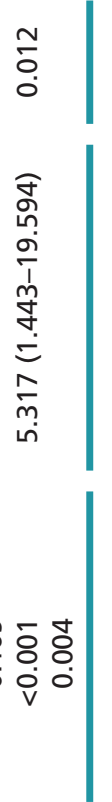

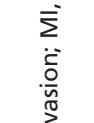

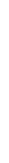

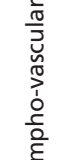

$\stackrel{\sum}{\grave{s}}$

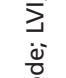

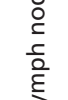

zi

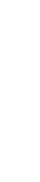

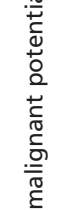

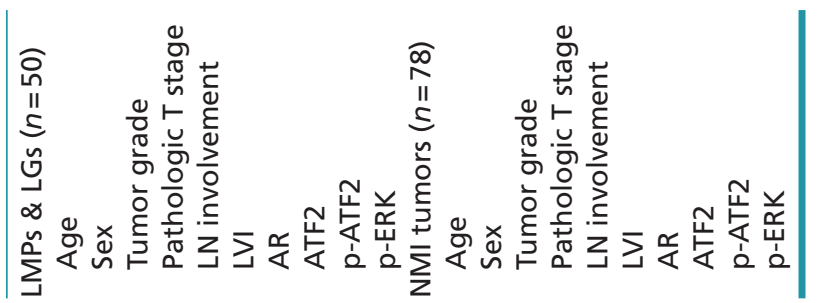


cell carcinoma of the skin (17), compared with respective normal/benign controls. In addition, ATF2 or p-ATF2 overexpression in breast cancer tissues was shown to associate with lower disease-free survival $(P=0.079)$ or higher overall survival $(P=0.047)$ rates, respectively (30). Using IHC in our bladder TMAs, we found significant increases in the expression levels of ATF2, p-ATF2 and p-ERK in bladder cancers, compared with corresponding non-neoplastic urothelial tissues. ATF2 expression was also elevated in high-grade and muscle-invasive bladder cancers, compared with low grade and non-muscle-invasive tumors, respectively. Prognostic analysis of the expression of these proteins further revealed that overexpression of ATF2, p-ATF2 and p-ERK was an independent predictor of recurrence of low grade tumors, cancer-specific mortality of muscle-invasive tumors and disease progression of muscle-invasive tumors, respectively. However, it was unexpected that some of the known risk factors (e.g. pTa vs pT1 in non-muscle-invasive tumors, pN0 vs pN1-3 in muscle-invasive tumors) were not significantly associated with clinical outcomes in the present study, possibly due to limited numbers of patients in each group and lack of statistical power. Meanwhile, there were significant positive correlations between the expression levels of p-ATF2 and p-ERK, ATF2 and AR (high-grade non-muscleinvasive tumors only) and p-ATF2 and AR. Current immunohistochemical findings in 129 bladder tumors thus support our in vitro/in vivo data suggesting that activation of ATF2 signals contributes to the induction of urothelial tumorigenesis and tumor progression.

In conclusion, ATF2 appeared to be activated in bladder cancer, which was associated with tumor development and progression. In particular, p-ATF2 expression in muscle-invasive bladder cancers may serve as a reliable prognosticator. ATF was also found to involve the regulation of urothelial tumorigenesis as well as tumor growth in the presence of activated AR, while androgen could activate ATF2 in bladder cancer cells. Accordingly, ATF2 inhibition, together with AR inactivation especially in AR-positive cases, has the potential of being an effective chemopreventive and/or therapeutic approach for urothelial carcinoma.

\section{Supplementary data}

This is linked to the online version of the paper at https://doi.org/10.1530/ EC-18-0364.

\section{Declaration of interest}

The authors declare that there is no conflict of interest that could be perceived as prejudicing the impartiality of the research reported.

\section{Funding}

The research did not receive any specific grant from any funding agency in the public, commercial, or not-for-profit sector.

\section{Author contribution statement}

Concept and design of experiments: $\mathrm{S} \mathrm{I}$ and $\mathrm{H} \mathrm{M}$; Acquisition of data: S I, T M, H I, G J, T G, Y N, and G J N; Analysis and interpretation of data: $S I$ and $H$ M; Writing and review of the manuscript: S I and H M; Supervision of the work: $\mathrm{H} \mathrm{M}$.

\section{References}

1 Torre LA, Bray F, Siegel RL, Ferlay J, Lortet-Tieulent J \& Jemal A. Global cancer statistics, 2012. CA: A Cancer Journal of Clinicians 2015 65 87-108. (https://doi.org/10.3322/caac.21262)

2 Inoue S, Mizushima T \& Miyamoto H. Role of the androgen receptor in urothelial cancer. Molecular and Cellular Endocrinology 2018465 73-81. (https://doi.org/10.1016/j.mce.2017.06.021)

3 Kashiwagi E, Ide H, Inoue S, Kawahara T, Zheng Y, Reis LO, Baras AS $\&$ Miyamoto $\mathrm{H}$. Androgen receptor activity modulates responses to cisplatin treatment in bladder cancer. Oncotarget 20167 49169-49179. (https://doi.org/10.18632/oncotarget.9994)

4 Ide H, Inoue S, Mizushima T, Jiang G, Chuang KH, Oya M \& Miyamoto $\mathrm{H}$. Androgen receptor signaling reduces radiosensitivity in bladder cancer. Molecular Cancer Therapeutics 201817 1566-1574. (https://doi.org/10.1158/1535-7163.MCT-17-1061)

5 Angel P \& Karin M. The role of Jun, Fos and the AP-1 complex in cell-proliferation and transformation. Biochimica et Biophysica Acta 1991 1072 129-157. (https://doi.org/10.1016/0304-419X(91)90011) 6 Lopez-Bergami P, Lau E \& Ronai Z. Emerging roles of ATF2 and the dynamic AP1 network in cancer. Nature Reviews Cancer 201010 65-76. (https://doi.org/10.1038/nrc2681)

7 Ouwens DM, de Ruiter ND, van der Zon GC, Carter AP, Schouten J, van der Burgt C, Kooistra K, Bos JL, Maassen JA \& van Dam H. Growth factors can activate ATF2 via a two-step mechanism: phosphorylation of Thr71 through the Ras-MEK-ERK pathway and of Thr69 through RalGDS-Src-p38. EMBO Journal 200221 3782-3793. (https://doi.org/10.1093/emboj/cdf361)

8 Zheng Y, Izumi K, Yao JL \& Miyamoto H. Dihydrotestosterone upregulates the expression of epidermal growth factor receptor and ERBB2 in androgen receptor-positive bladder cancer cells. EndocrineRelated Cancer 201118 451-464. (https://doi.org/10.1530/ERC-110010)

9 Kawahara T, Shareef HK, Aljarah AK, Ide H, Li Y, Kashiwagi E, Netto GJ, Zheng Y \& Miyamoto H. ELK1 is up-regulated by androgen in bladder cancer cells and promotes tumor progression. Oncotarget $2015629860-29876$.

10 Miyamoto H, Yang Z, Chen YT, Ishiguro H, Uemura H, Kubota Y, Nagashima Y, Chang YJ, Hu YC, Tsai MY, et al. Promotion of bladder cancer development and progression by androgen receptor signals. Journal of the National Cancer Institute 200799 558-568. (https://doi. org/10.1093/jnci/djk113)

11 Inoue S, Ide H, Mizushima T, Jiang G, Netto GJ, Gotoh M \& Miyamoto H. Nuclear factor- $\kappa \mathrm{B}$ promotes urothelial tumorigenesis and cancer progression via cooperation with androgen receptor signaling. Molecular Cancer Therapeutics 201817 1303-1314. (https:// doi.org/10.1158/1535-7163.MCT-17-0786)

12 Kashiwagi E, Inoue S, Mizushima T, Chen J, Ide H, Kawahara T, Reis LO, Baras AS, Netto GJ \& Miyamoto H. Prostaglandin receptors induce urothelial tumourigenesis as well as bladder cancer progression and cisplatin resistance presumably via modulating PTEN expression. British Journal of Cancer 2018118 213-223. (https://doi.org/10.1038/bjc.2017.393)

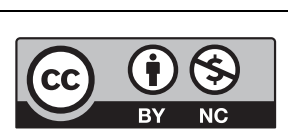

This work is licensed under a Creative Commons Attribution-NonCommercial 4.0 International License. 
13 Izumi K, Zheng Y, Hsu JW, Chang C \& Miyamoto H. Androgen receptor signals regulate UDP-glucuronosyltransferases in the urinary bladder: a potential mechanism of androgen-induced bladder carcinogenesis. Molecular Carcinogenesis 201352 94-102. (https://doi. org/10.1002/mc.21833)

14 Reznikoff CA, Loretz LJ, Christian BJ, Wu SQ \& Meisner LF. Neoplastic transformation of SV40-immortalized human urinary tract epithelial cells by in vitro exposure to 3-methylcholanthrene. Carcinogenesis 19889 1427-1436. (https://doi.org/10.1093/ carcin/9.8.1427)

15 Miyamoto H, Yao JL, Chaux A, Zheng Y, Hsu I, Izumi K, Chang C, Messing EM, Netto GJ \& Yeh S. Expression of androgen and oestrogen receptors and its prognostic significance in urothelial neoplasm of the urinary bladder. BJU International 2012109 1716-1726. (https://doi.org/10.1111/j.1464-410X.2011.10706.x)

16 Hsu CC \& Hu CD. Critical role of N-terminal end-localized nuclear export signal in regulation of activating transcription factor 2 (ATF2) subcellular localization and transcriptional activity. Journal of Biological Chemistry 2012287 8621-8632. (https://doi.org/10.1074/ jbc.M111.294272)

17 Papassava P, Gorgoulis VG, Papaevangeliou D, Vlahopoulos S, van Dam H \& Zoumpourlis V. Overexpression of activating transcription factor-2 is required for tumor growth and progression in mouse skin tumors. Cancer Research 200464 8573-8584. (https://doi. org/10.1158/0008-5472.CAN-03-0955)

18 Chen SY, Takeuchi S, Moroi Y, Hayashida S, Kido M, Tomoeda T, Uenotsuchi T, Tu YT, Furue M \& Urabe K. Concordant overexpression of phosphorylated ATF2 and STAT3 in extramammary Paget's disease. Journal of Cutaneous Pathology 200936 402-408. (https://doi. $\operatorname{org} / 10.1111 / \mathrm{j} .1600-0560.2008 .01076 . x)$

19 Duffey D, Dolgilevich S, Razzouk S, Li L, Green R \& Gorti GK. Activating transcription factor-2 in survival mechanisms in head and neck carcinoma cells. Head and Neck 201133 1586-1599. (https:// doi.org/10.1002/hed.21648)

20 Su L, Sampaio AV, Jones KB, Pacheco M, Goytain A, Lin S, Poulin N, Yi L, Rossi FM, Kast J, et al. Deconstruction of the SS18-SSX fusion oncoprotein complex: Insight into disease etiology and therapeutics. Cancer Cell 201221 333-347. (https://doi.org/10.1016/j. ccr.2012.01.010)

21 Li M, Wu X, Liu N, Li X, Meng F \& Song S. Silencing of ATF2 inhibits growth of pancreatic cancer cells and enhances sensitivity to chemotherapy. Cell Biology International 201741 599-610. (https:// doi.org/10.1002/cbin.10760)

22 Woo IS, Kohno T, Inoue K, Ishii S \& Yokota J. Infrequent mutations of the activating transcription factor- 2 gene in human lung cancer, neuroblastoma and breast cancer. International Journal of Oncology 200220 527-531. (https://doi.org/10.3892/ijo.20.3.527)

23 Song H, Ki SH, Kim SG \& Moon A. Activating transcription factor 2 mediates matrix metalloproteinase- 2 transcriptional activation induced by p38 in breast epithelial cells. Cancer Research $2006 \mathbf{6 6}$ 10487-10496. (https://doi.org/10.1158/0008-5472.CAN-06-1461)

24 Maekawa T, Sano Y, Shinagawa T, Rahman Z, Sakuma T, Nomura S, Licht JD \& Ishii S. ATF-2 controls transcription of Maspin and GADD45 alpha genes independently from p53 to suppress mammary tumors. Oncogene 200827 1045-1054. (https://doi.org/10.1038/ sj.onc.1210727)

25 Bhoumik A, Fichtman B, Derossi C, Breitwieser W, Kluger HM, Davis S, Subtil A, Meltzer P, Krajewski S, Jones N, et al. Suppressor role of activating transcription factor 2 (ATF2) in skin cancer. PNAS 2008105 1674-1679. (https://doi.org/10.1073/ pnas.0706057105)

26 Lau E, Kluger H, Varsano T, Lee K, Scheffler I, Rimm DL, Ideker T $\&$ Ronai ZA. PKC $\varepsilon$ promotes oncogenic functions of ATF2 in the nucleus while blocking its apoptotic function at mitochondria. Cell 2012148 543-555. (https://doi.org/10.1016/j.cell.2012.01.016)

27 Liu S, Wang F, Yan L, Zhang L, Song Y, Xi S, Jia J \& Sun G. Oxidative stress and MAPK involved into ATF2 expression in immortalized human urothelial cells treated by arsenic. Archives of Toxicology 2013 87 981-989. (https://doi.org/10.1007/s00204-013-1058-9)

28 Hamdi MM \& Mutungi G. Dihydrotestosterone stimulates amino acid uptake and the expression of LAT2 in mouse skeletal muscle fibres through an ERK1/2-dependent mechanism. Journal of Physiology 2011589 3623-3640. (https://doi.org/10.1113/ jphysiol.2011.207175)

29 Ricote M, García-Tuñón I, Bethencourt F, Fraile B, Onsurbe P, Paniagua R \& Royuela M. The p38 transduction pathway in prostatic neoplasia. Journal of Pathology 2006208 401-407. (https://doi. org/10.1002/path.1910)

30 Knippen S, Löning T, Müller V, Schröder C, Jänicke F \& Milde-Langosch K. Expression and prognostic value of activating transcription factor 2 (ATF2) and its phosphorylated form in mammary carcinomas. Anticancer Research 200929 183-189.

Received in final form 19 October 2018

Accepted 9 November 2018 https://ec.bioscientifica.com

https://doi.org/10.1530/EC-18-0364 (c) 2018 The authors Published by Bioscientifica Ltd

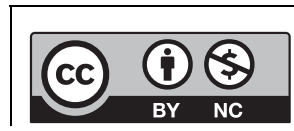

This work is licensed under a Creative Commons Attribution-NonCommercial 4.0 International License. 